\title{
THE FUNCTION OF APICAL ECTODERMAL RIDGE IN THE FORMATION OF LIMB
}

\author{
Avijit Mallick ${ }^{1}$ \\ Department of Zoology, University of Melbourne Melbourne, Victoria, Australia
}

For the past couple of decades, scientists have pondered over the question of how body structures of various sizes and shapes are formed; the vertebrate limb was a wonderful model system to address their questions. Many challenges were faced when trying to understand how the embryonic bud, containing only homogenous mesenchymal cells and epithelium, develops into an organ with numerous diverse forms. More than 50 years ago, an experiment performed by John W. Saunders identified the ectoderm rimming the distal tip of the limb bud (apical ectodermal ridge, AER) as a source of signals essential for limb development (Saunders 1948). The AER and the zone of polarizing activity (ZPA) are the two key signaling centers that coordinate proximodistal and anterioposterior limb development (Yu K 2008). The specialized ectodermal structure at the dorsal tip of the limb bud called the AER plays a crucial role in proximodistal patterning, which has been proved by skeletal truncation after surgical removal of AER. The AER functions by sending signals to the adjacent mesenchyme via fibroblast growth factor (FGF), which has been identified as the main component of the AER signaling (Freeman 2000). FGFs elicit their function by binding and activating high affinity FGF receptors, a subclass of receptor tyrosine kinases. During limb development, the activated FGFRs trigger several signaling pathways such as the MAP (Mitogen-activated protein) kinase pathway in the mesenchymal cells adjacent to the AER (Ornitz and Itoh 2001, Zhang et al. 2006). AER-FGF signals regulate limb development by maintaining mesenchymal cell survival and in turn, maintaining the progenitor cell population needed for proximal-distal limb formation. In the current investigation, we utilized methods similar to those of John Saunders and examined the function of AER more closely.

A small hole, $2 \mathrm{~cm}$ in diameter was cut in the blunt end of a 3 day-old-egg using crunching forceps. The egg shell membrane was then removed using fine pair of forceps. Following piercing of the vitelline membrane, the visible chicken embryo was examined under a dissecting microscope to determine its Hamburger and Hamilton (HH)* stage. To avoid bacterial infection and drying out, an antibiotic saline was added to the embryo. Finally, the ectoderm overlying the limb bud (either fore or hind limb) of 100 embryos were removed and incubated for a week after sealing the egg. Two other controls of 100 embryos each were carried out as follows: (a) Operational control ('sham' operation where the ectoderm was scratched but not removed) and (b) experimental control (nothing was done to this embryo). After a week of incubation in the incubator at 211.1 degrees Fahrenheit and 50\% humidity, the surviving embryos in all conditions were examined to check the $\mathrm{HH}$ stage reached and the embryonic limb development.

${ }^{1}$ Present address: Doctor's villa, Nirapad H/S, Dewan Bajar, West Bakalia, Chiitagong, Bangladesh.

<avijitsunshine@gmail.com>.

*The operational stages are described as Hamburger-Hamilton stages (HH) which are a series of 46 chronological stages in chick development, starting from laying of the egg and ending with a newly hatched egg. 


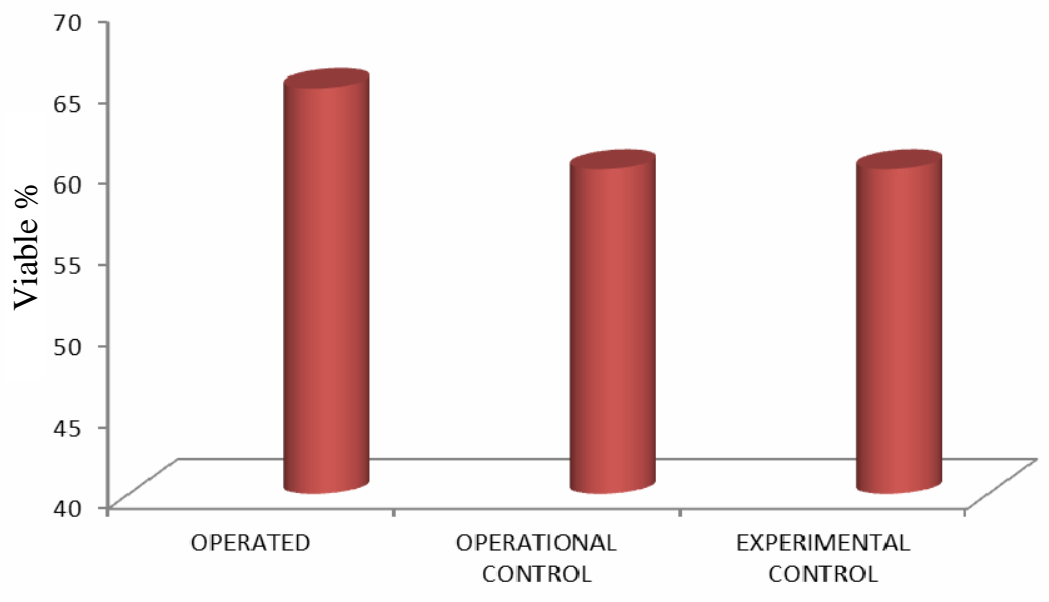

Description of sample

Fig. 1. The percentage of embryos surviving after one week of incubation for each of the three different samples. In each sample, the percentages of viable embryos were within $5 \%$ of one other, thus having no influence on the viability of embryos in our experiment.

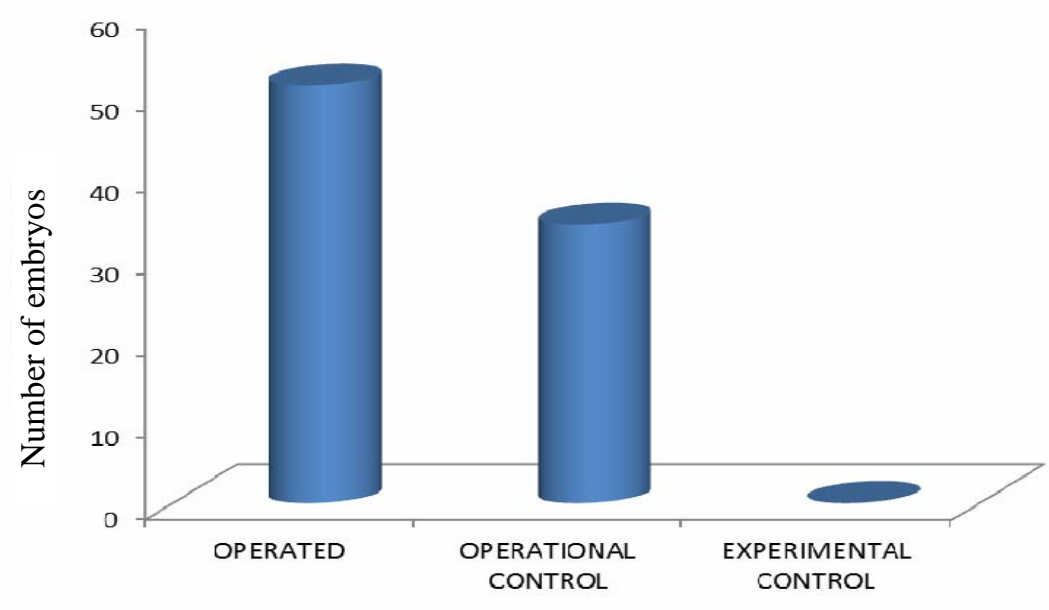

Description of sample

Fig. 2. The number of embryos with a defective limb in each sample of 100 . The sham control which had the ectoderm scratched also gave rise to embryos with a defective limb.

A Chi square test was carried out to check if there was a significant difference in the number of embryos with defective limbs in each of the samples. The number of embryos in each of the samples was 100 and the numbers of embryos with the defective limb formation were as follows: 51 for the operated sample, 34 for the 'sham' sample and 0 for the control sample. The null hypothesis to be tested was whether the difference between the numbers of embryos with the 
defective limb formation in each sample was due to chance or not. The Chi square test gave a $\mathrm{p}<$ 0.05 (degree of freedom of 2) which rejected the null hypothesis and confirmed that the difference in the number of embryos was not due to chance.

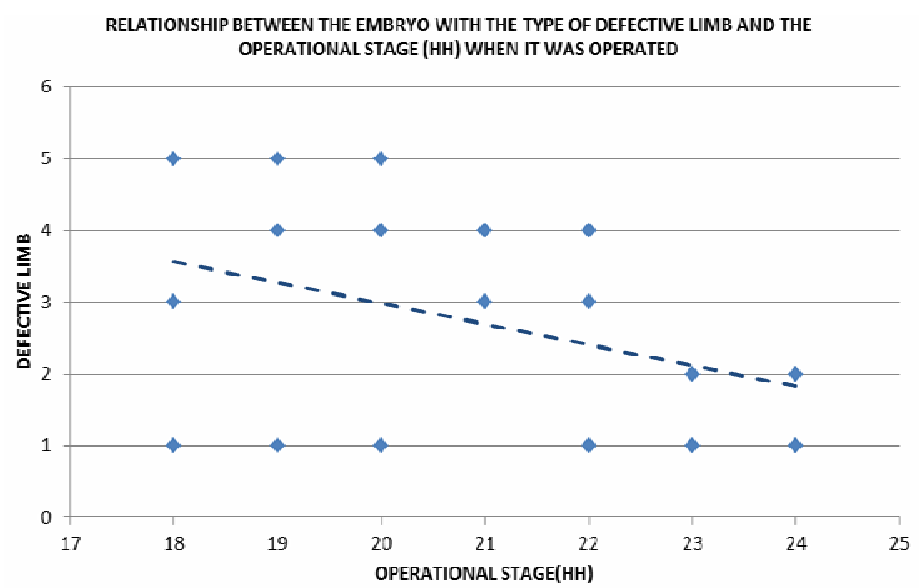

Fig. 3. The correlation between the limb defect and the HH stage of operation is shown here, eliminating any normal embryos. This graph has a trend line demonstrating that the limb malformation moves towards the distal axis with a later HH stage operation. 0. Normal. 1. Digit defect. 2. Defect at wrist. 3. Defect of knee. 4. Defect of upper arm. 5. Defect of shoulder.

In the present experiment, the operation did not affect the rate of death of the embryos as shown in Fig. 1, which could have otherwise affected the experiment's validity. The percentage of viable embryos for each of the samples (control, operated and sham) was very close (around 60\%). However, the number of embryos with limb malformations was different between the three samples: being highest (51) for the operated embryos and lowest (0) for the control embryos, as shown in Fig. 2. A Chi-square test demonstrated that the differences in limb formation between AER operated samples were statistically significant, suggesting the importance of AER in limb development.

The specialized ectodermal structure at the dorsal tip of the limb bud, called the apical ectodermal ridge (AER), plays a crucial role in proximodistal patterning. Our results support the importance of AER in the limb development as seen in Fig. 4, where skeletal truncation results from surgical removal of AER. Fibroblast growth factors (FGFs) released by the AER are necessary for proximodistal limb formation. Fgf4 and Fgf8 are the two most important FGFs among four that are necessary for the skeletal formation of limb, as disruption of these genes lead to a complete failure of limb formation (Boulet et al. 2004, Sun et al. 2002).

Knocking out both Fgf4 and Fgf8 produced a more severe phenotype than knocking out Fgf8 alone. AER-FGF signaling ensures that there are enough progenitor cells required for the normal limb development (Mariani et al. 2008). The function of FGF protein becomes clear considering that skeletal elements are rescued when FGF beads are added to the limb bud lacking the AER. In 
Saunder's study (1948), removal of AER at an early limb bud stage leads only to the formation of the proximal segment (upper arm, thigh) leaving the middle (fore arm, lower leg) and distal (wrist and hand, ankle and foot) segment. However, when the AER was removed at a later HH stage, only the autopod (wrist and hand, ankle and foot) remained undeveloped (Sun et al. 2002, Cooper et al. 2011). This observation matches our experiment's results as shown in Fig. 4. When operated at a very early $\mathrm{HH}$ stage of 17 , most of the limb phenotype was 5 (defect at the shoulder), compared to mainly phenotype 2 (defect at the wrist) when operated at HH stage 23. The results suggested a presence of relationship between the limb phenotype and the operational stage, which supports the findings of John Saunder (Saunders 1948).

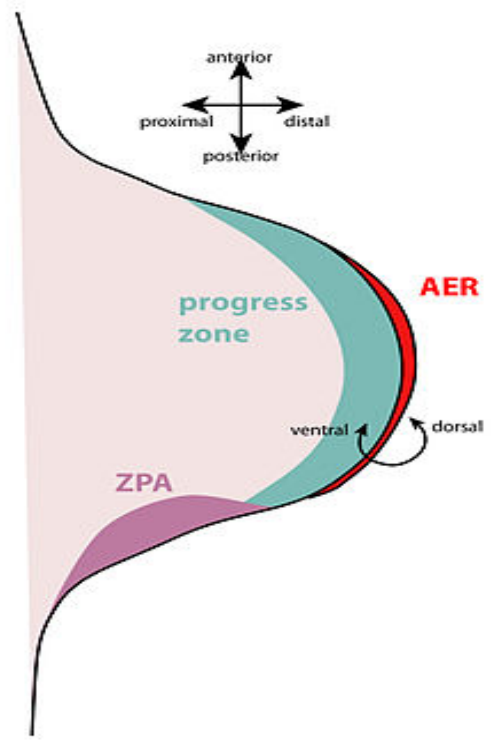

Fig. 4. Demonstrates the position of AER (apical ectodermal ridge), ZPA (zone of polarizing activity) and progress zone in the limb bud. FGF8 secreted from the AER maintains the mesenchymal cells in the progress zone.

Two models have been proposed to explain the proximal-distal (PD) axis patterning of the limb (1). The progress zone model states that a cell's fate depends on the amount of time it spends in the progress zone (PZ) (2). The early allocation or progenitor expansion model states that the instructive signals from the AER are responsible for PD axis formation (Sun et al. 2002). The progress zone model does not seem to be in accordance with the previous research findings. The model states that progenitor cells in the progress zone become progressively distalized and cease distalizing as they leave the progress zone. However, it has previously been shown that FGF beads rescue distal limb elements even after AER removal, which does not support the PZ model. Our experiment found that operation at a later $\mathrm{HH}$ stage have led to more distal limb defects, which also suggests that progress zone cells continue to distalize, as regulated by AER-FGF signaling (Expansion model) (Niswander et al. 1993, Fallon et al. 1994). 
In summary, FGF signals from the AER directly or indirectly regulate mesenchymal survival, proliferation and differentiation during development. Our results demonstrated that the AER plays an important role in limb formation in chicken embryos. Although we did not study the signaling pathways involved in limb formation, we were able to show that the AER was responsible for limb formation. This was evident because there was a significant difference between the number of embryos with defective limbs in the operational control and normal sample compared to the operated sample. Moreover, we demonstrated that the removal of AER at a later HH stage leads to distal limb defect which supports the expansion model.

\section{Acknowledgement}

The author would like to specially thank Robb de Longh, Associate Professor from the Department of Anatomy and Neuroscience, University of Melbourne for his help in carrying out the experiment and interpretation of data.

\section{References}

Boulet, A. M., A.M. Moon, B.R. Arenkiel and M.R. Capecchi. 2004. The roles of Fgf4 and Fgf8 in limb bud initiation and outgrowth. Dev. Biol. 273: 361-372. (doi: 10.1016/j.ydbio.2004.06.012)

Cooper KL, J.K. Hu , D. ten Berge, M. Fernandez-Teran, M.A. Ros, and C.J. Tabin. 2011. Initiation of proximal-distal patterning in the vertebrate limb by signals and growth. Science 332(6033): 1083-6. (doi: 10.1126/science1199499)

Fallon, J. et al. 1994. FGF-2: Apical ectodermal ridge growth signal for chick limb development. Science 264: 104-107. (doi: 10.1126/science.7908145)

Freeman, M. 2000. Feedback control of intracellular signaling in development. Nature 408: 313-319. (doi: $10.1038 / 35042500)$

Mariani F.V., C.P. Ahn and G.R. Martin. 2008. Genetic evidence that FGFs have an instructive role in limb proximaldistal patterning. Nature 453(7193): 401-5. doi: 10.1038/nature06876)

Niswander, L., C. Tickle, A. Vogel, I. Booth and G.R. Martin. 1993. FGF-4 replaces the apical ectodermal ridge and directs outgrowth and patterning of the limb. Cell 75: 579-587. (doi: 10.1016/00928674(93)90391-3)

Ornitz, D.M. and N. Itoh. 2001. Fibroblast growth factors. Genome Biol. 2: reviews 3005.1-3005.2. (doi: 10.1186/gb-2001-2-3-reviews30005)

Saunders, J.W. Jr. 1948. The proximo-distal sequence of the origin of the parts of the chick wing and the role of the ectoderm. J. Exp. Zool. 108: 363-403. (doi: 10.1002/jez.1401080304)

Sun, X., F.V. Mariani and G.R. Martin. 2002. Functions of FGF signalling from the apical ectodermal ridge in limb development. Nature 418: 501-508. (doi: 10.1038/nature00902)

Yu, K. and D.M Ornitz. 2008. FGF signaling regulates mesenchymal differentiation and keletal patterning along the limb bud proximo-distal axis. Development 135: 483-91. doi: 10. 1242/dev.013268)

Zhang, X., O.A. Ibrahimi, S.K. Olsen, H. Umemori, M. Mohammadi and D.M. Ornitz. 2006. Receptor specificity of the fibroblast growth factor family. The complete mammalian FGF family. J. Biol. Chem. 281: 15694-15700. (doi: 10.1074/jbc.M601252200)

(Manuscript received on 30 October, 2013; revised on 21 November, 2013) 